\title{
A theoretical scenario for PMS $\delta$ Scuti stars
}

\author{
A. Ruoppo, ${ }^{1,2}$ M. Marconi, ${ }^{2}$ M. Marques, ${ }^{3,4,5}$ M. J. P. F. G. Monteiro, ${ }^{4,5}$ \\ J. Christensen-Dalsgaard, ${ }^{6}$ F. Palla, ${ }^{7}$ V. Ripepi ${ }^{2}$ \\ ${ }^{1}$ Dipartimento di Scienze Fisiche, Università Federico II, Napoli, Italy \\ ${ }^{2}$ INAF-Osservatorio Astronomico di Capodimonte, Napoli, Italy \\ ${ }^{3}$ Universidade de Coimbra, Departamento de Matemática - FCTUC, Portugal \\ ${ }^{4}$ Departamento de Matemática Aplicada, Universidade do Porto, Portugal \\ ${ }^{5}$ Centro de Astrofísica da Universidade do Porto, Portugal \\ ${ }^{6}$ DASC and Institut for Fysik og Astronomi, Aarhus Universitet, Denmark \\ ${ }^{7}$ INAF-Osservatorio Astrofisico di Arcetri, Firenze, Italy
}

\begin{abstract}
Nonradial pulsation models have been computed by means of the Aarhus adiabatic code along an extensive set of CESAM PMS evolutionary tracks. A theoretical tool for the interpretation of observed periodicities is proposed.
\end{abstract}

\section{Introduction}

Pre-Main sequence (PMS) $\delta$ Scuti stars are intermediate mass stars that cross the pulsation instability strip of more evolved classical pulsators during their evolution towards the Main Sequence. The number of discovered pulsating PMS stars is growing, but only a few stars have been studied in detail. As a consequence, the overall properties of this class of variables are still poorly determined. In this context we present our first results based on an extensive grid of evolutionary PMS models computed using the CESAM (Morel 1997) code to which the Aarhus adiabatic code (http://astro.phys.au.dk/ jcd/adipack.n/) is applied for the determination of the frequencies. This method can be in principle applied to other classes of pulsating stars and is expected to work best for variables pulsating in a large number of modes in the asymptotic frequency regime.

\section{A method to reproduce observed frequencies}

The steps we follow to compare the observed pulsation frequencies with the theoretical ones are: (1) to determine a range in mass and in the expected large frequency separation $(\Delta \nu)$ based on the available estimates of luminosity and effective temperature; (2) to estimate $\Delta \nu$ from the frequency data; (3) to reduce the mass range by using the observed $\Delta \nu$; (4) to compare the predicted frequencies with the observed ones in the echelle diagram for the selected models; (5) to provide the best fit model stellar parameters and a mode identification. Further details can be found in Ruoppo et al. (2007). The predictive capabilities of the method are tested by applying it to a test star (star1), that is a PMS stellar model computed using the STAROX code (Roxburgh 2005, http://www . astro.up.pt/corot/) with the pulsation radial and non radial frequencies computed using the POSC code (Monteiro 1996). Application of our procedure allows us to define the following ranges in mass and $\Delta \nu$ for star1: $1.6<$ $M / M_{\odot}<3.4,20 \mu \mathrm{Hz}<\Delta \nu<80 \mu \mathrm{Hz}$. We compared the simulated frequencies with the theoretical ones in the echelle diagram by varying $\Delta \nu$ in the range determined above. The best agreement (see Fig. 1) is obtained for a PMS model with $M=2 M_{\odot}, T_{\text {eff }}=8184 \mathrm{~K}$. The obtained mass coincides with the true one whereas the effective temperature differs from 


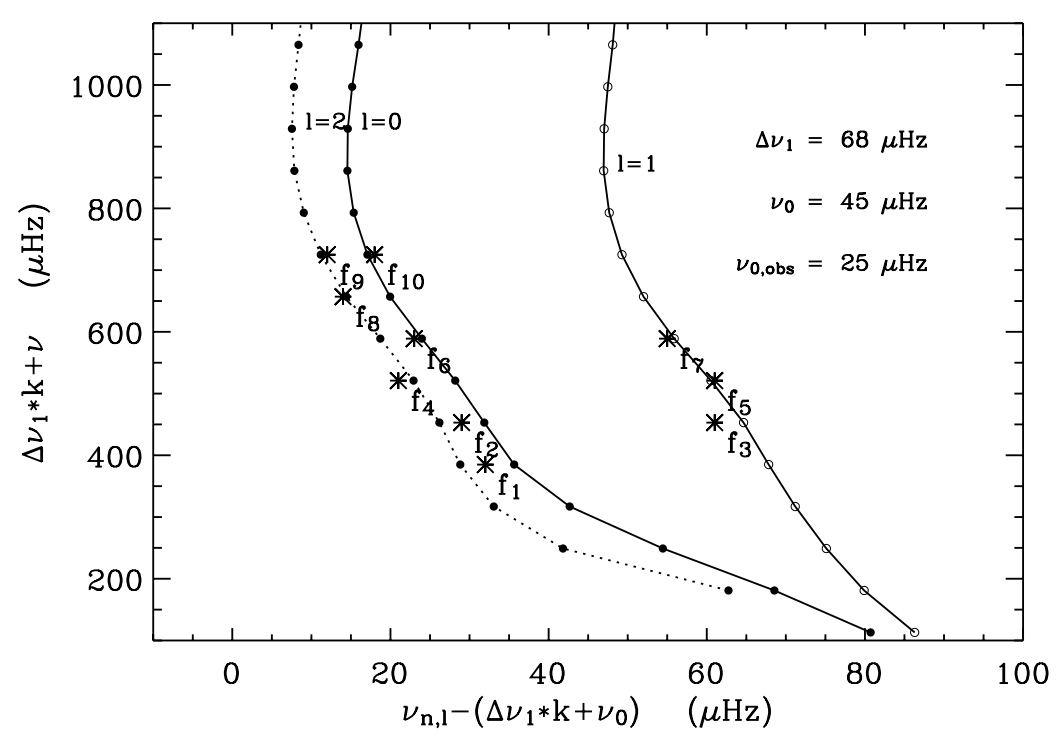

Figure 1: The best echelle diagram for Star1: the harmonic degree $\ell$ varies from 0 to 2 while the radial order ranges from 1 to 15 .

the true one by less than $250 \mathrm{~K}$. Moreover, our best-fit model reproduces all the observed frequencies, with the correct mode identification.

We note that we have not taken into account rotation that is expected to change the identification obtained for non-radial modes and shift the frequencies (more detail can be found in Ripepi et al. 2007 and Ruoppo et al. 2007).

Acknowledgments. We thank the anonymous referee for valuable comments.

\section{References}

Monteiro M. J. P. F. G., 1996, PhD Thesis, Queen Mary College, Univ. of London, UK

Morel P., 1997, A\&AS, 124, 597

Ripepi V., Bernabei S., Marconi M., et al., 2007, A\&A, 462, 1023

Ruoppo A., Marconi M., Marques M., et al., 2007, A\&A, in press 